\title{
HispanismeS
}

Revue de la Société des Hispanistes Français

18 | 2021

Murs, barrières, obstacles dans les mondes hispaniques II

\section{Ampparito ou l'art de déconcerter le passant}

Ampparito or the art of confusing the passer-by

Ampparito o el arte de descolocar al viandante

\section{Anne Puech}

\section{OpenEdition}

Journals

Édition électronique

URL : https://journals.openedition.org/hispanismes/14078

DOI : 10.4000/hispanismes. 14078

ISSN : 2270-0765

\section{Éditeur}

Société des Hispanistes Français

\section{Référence électronique}

Anne Puech, «Ampparito ou l'art de déconcerter le passant », HispanismeS [En ligne], 18| 2021, mis en ligne le 31 décembre 2021, consulté le 30 janvier 2022. URL : http://journals.openedition.org/ hispanismes/14078; DOI : https://doi.org/10.4000/hispanismes.14078

Ce document a été généré automatiquement le 30 janvier 2022.

\section{(c) $(1) \Theta \Theta$}

Les contenus de cette revue sont mis à disposition selon les termes de la Licence Creative Commons Attribution - Pas d'Utilisation Commerciale - Pas de Modification 4.0 International. 


\title{
Ampparito ou l'art de déconcerter le passant
}

\author{
Ampparito or the art of confusing the passer-by \\ Ampparito o el arte de descolocar al viandante
}

Anne Puech

\section{Introduction}

L'utilisation du prénom Amparo, de surcroît, dans sa version hypocoristique, pourrait nous laisser penser que l'artiste qui nous intéresse ici est une femme d'un certain âge, qui, pour tromper l'ennui, aurait décidé de pimenter son quotidien en s'adonnant à des activités picturales illégales dans l'espace public. Or, il s'agit d'un homme, diplômé de la faculté des Beaux-Arts de l'université Complutense de Madrid en 2014¹. Comme il l'a expliqué au journaliste Enrique Alpañés, qui lui a consacré un article en janvier 2020, Ampparito est un nom de scène trompeur. S'il a choisi ce pseudonyme, c'est pour créer un contraste avec les signatures répandues dans l'espace public, des noms choisis dans une logique de compétition, des pseudonymes «cool, qui accrochent ${ }^{2}$ ». Ses interventions, tout comme son nom de scène, ont pour but de décontenancer le spectateur. Dès lors, la dimension ludique de son œuvre inviterait-elle le spectateur à reconsidérer la fonction du mur? C'est ce que nous proposons d'étudier ici, en nous appuyant sur différentes interventions réalisées par Ampparito dans l'espace public espagnol.

Avant de commencer cette étude, il nous semble important de proposer un cadrage conceptuel des principales pratiques picturales et scripturales réalisées dans l'espace public. Pour commencer, nous ne parlerons pas de graffiti (aussi appelé graffitisignature ou graffiti Hip Hop). Il relève, en effet, d'un fonctionnement différent des interventions qui nous intéressent ici. En effet, le graffiti et le tag reposent sur une logique cryptique, de reproduction en série d'une signature et de conquête symbolique de l'espace public. Les scripteurs restreignent l'intelligibilité de leurs interventions à un certain nombre d'initiés. Ce qui n'est pas le but des artistes publics - comme 
Ampparito - qui destinent, au contraire, leur production au plus grand nombre dans un souci de démocratisation de l'expérience esthétique, telle que l'avait pensée le philosophe John Dewey.

Par ailleurs, l'expression «d'art urbain » ne nous semble pas appropriée pour désigner ce type de pratique picturale car elle exclut, de fait, toutes les interventions artistiques réalisées en dehors des villes. Or, les peintures murales ont, de tout temps, pris place autant dans des zones urbaines que rurales. Cet article propose, d'ailleurs, une sélection d'œuvres réalisées aussi bien dans de grandes agglomérations que dans des villages.

La confusion vient probablement du fait que l'on assimile facilement peinture murale et graffiti et que ce dernier est, lui, intrinsèquement lié à la ville. On emploie donc aujourd'hui, à tort, l'expression d'art « urbain » pour désigner ces peintures singulières spontanées ou de commande alors qu'elles sont également réalisées dans des villages ou des zones non urbanisées.

Dans la mesure où nous étudions des interventions plastiques réalisées dans l'espace public, nous privilégions donc la locution d'«art public». Ce choix peut paraître étonnant, étant donné que l'expression fait référence, à l'origine, à des œuvres de commande dans l'espace public. Ces incursions de l'art sont à l'initiative d'une institution publique - la Mairie, l'État -, ou privée - une fondation, un propriétaire, un groupe religieux - afin d'installer une œuvre mémorielle, idéologique et/ou éducative. Mais un glissement sémantique a eu lieu et l'expression désigne, aujourd'hui, des interventions artistiques dont les auteurs travaillent dans le but de contribuer au bienêtre - intellectuel, visuel, esthétique, etc. - des spectateurs et estiment qu'ils réalisent une forme de service public. C'est pourquoi l'expression d'art "public» est à privilégier, en y accolant l'adjectif « indépendant » ou "spontanée » lorsqu'il s'agit d'une œuvre laissée sans autorisation, comme l'avait suggéré le chercheur Javier Abarca ${ }^{3}$.

\section{Porosité des espaces public et privé}

Ampparito, une fois diplômé des Beaux-Arts, a choisi de peindre dans la rue car cela lui permettait de régler deux problèmes majeurs : celui du stockage des toiles et celui de l'audience. Il pouvait atteindre un public conséquent et, de surcroit, observer discrètement la réaction des passants face à ses œuvres sans se soucier de l'accumulation des toiles dans son appartement en colocation.

Il aime aussi intervenir dans la rue plutôt que sur une toile, parce que cet espace multiplie les chances de «prendre le spectateur à l'improviste, de le déconcerter $»^{4}$. Et le trouble opère sur différents terrains, puisqu'il poste ses propres interventions sur les réseaux sociaux et les accompagne d'explications assez fournies, ajoutant encore une autre dimension à ses œuvres. Il occupe, de la sorte, plusieurs espaces d'exposition et atteint des publics variés. L'espace virtuel est intéressant, d'un point de vue artistique, car il constitue un terrain d'expérimentation et agit comme une caisse de résonnance. À travers ses œuvres, Ampparito cherche à interagir avec le public, il considère donc l'espace numérique comme une autre scène possible.

À la manière des artistes surréalistes, conceptuels ou Pop Art, il a pris comme sujet d'étude une série d'objets du quotidien qu'il a peints en grand format, dans l'espace public. Nous pouvons donner l'exemple de l'épingle à nourrice réalisée dans la ville 
française de Saint-Chamond, liée à l'activité sidérurgique. Voici les explications qu'il a fournies pour accompagner la peinture d'un serre-câble, réalisée dans un village de Castille :

Después del mejor festival en el que he estado, sufrí la peor resaca imaginable. Intentando hallar la mejor manera de gestionar semejante bajón, estuve reflexionando sobre compromisos y cómo de bueno sería si tuviese un trabajo decente, como funcionario por ejemplo y cualquier tipo de relación seria para aliviar esos días. En vez de esto, decidí pintar una brida de nylon en una casa con un hombre dentro ${ }^{5}$.

Figure 1. Ampparito, Sobre compromisos y abrazaderas, Castille, 2016

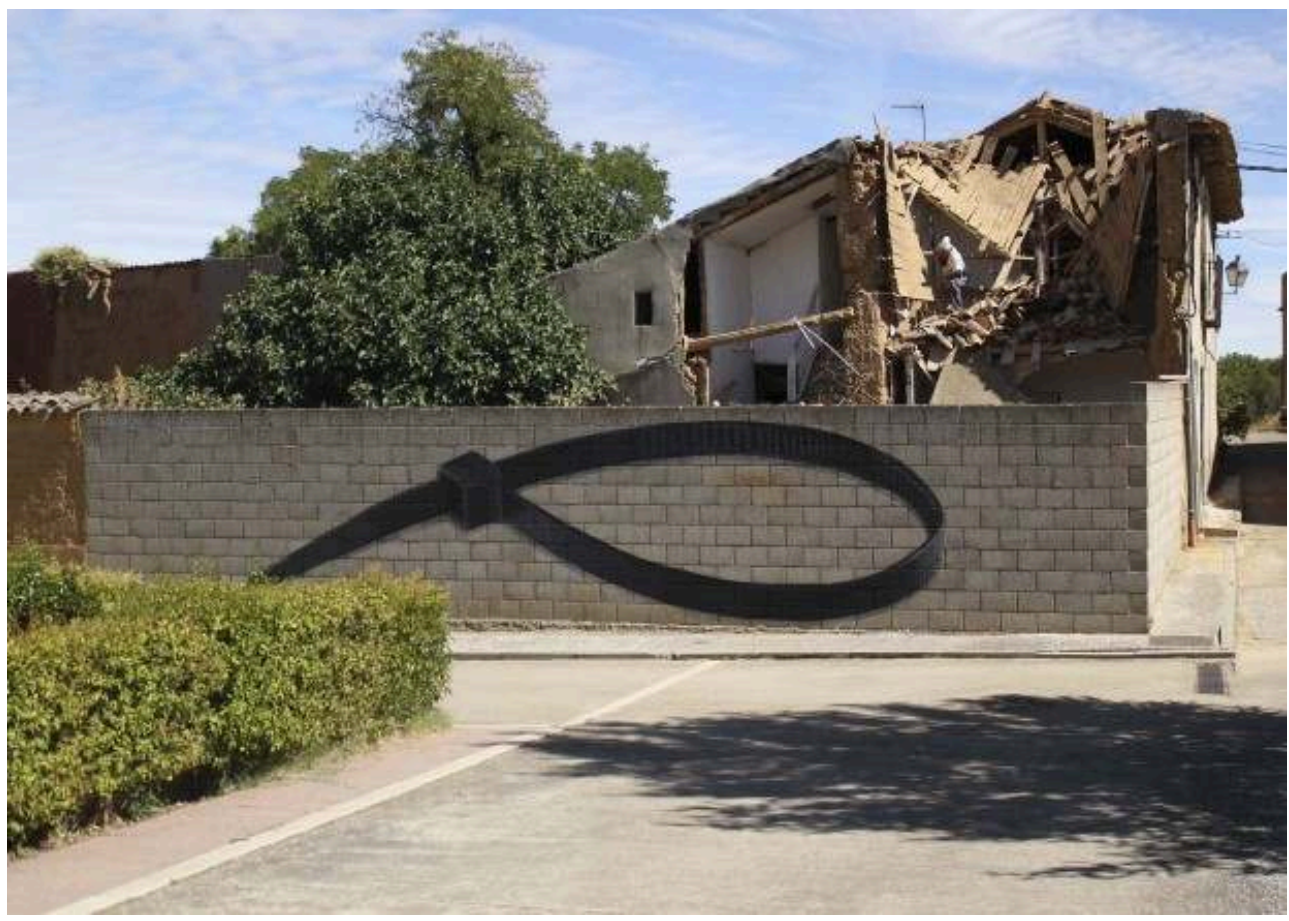

(c) Ampparito

On comprend qu'il ne se prend pas au sérieux et présente son travail avec un certain degré de dérision. Mais aussi qu'il fait du mur un espace de catharsis : il s'est engagé dans une voie professionnelle peu rassurante, celle d'artiste et a décidé de représenter ce à quoi il a "échappé ». Ce serre flex peut être interprété, à la lecture des mots d'Ampparito, comme une représentation du travail de fonctionnaire, pérenne, certes, mais monotone.

Les habitants de Bolaños de Campos, petit village de la province de Valladolid, ont probablement dû être interloqués lorsqu'ils ont découvert ce bouchon de stylo géant peint sur le mur d'un bâtiment agricole. 
Figure 2. Ampparito, Sobre la ansiedad, la incertidumbre y el rechinar de dientes, Becilla de Valderaduey, 2016

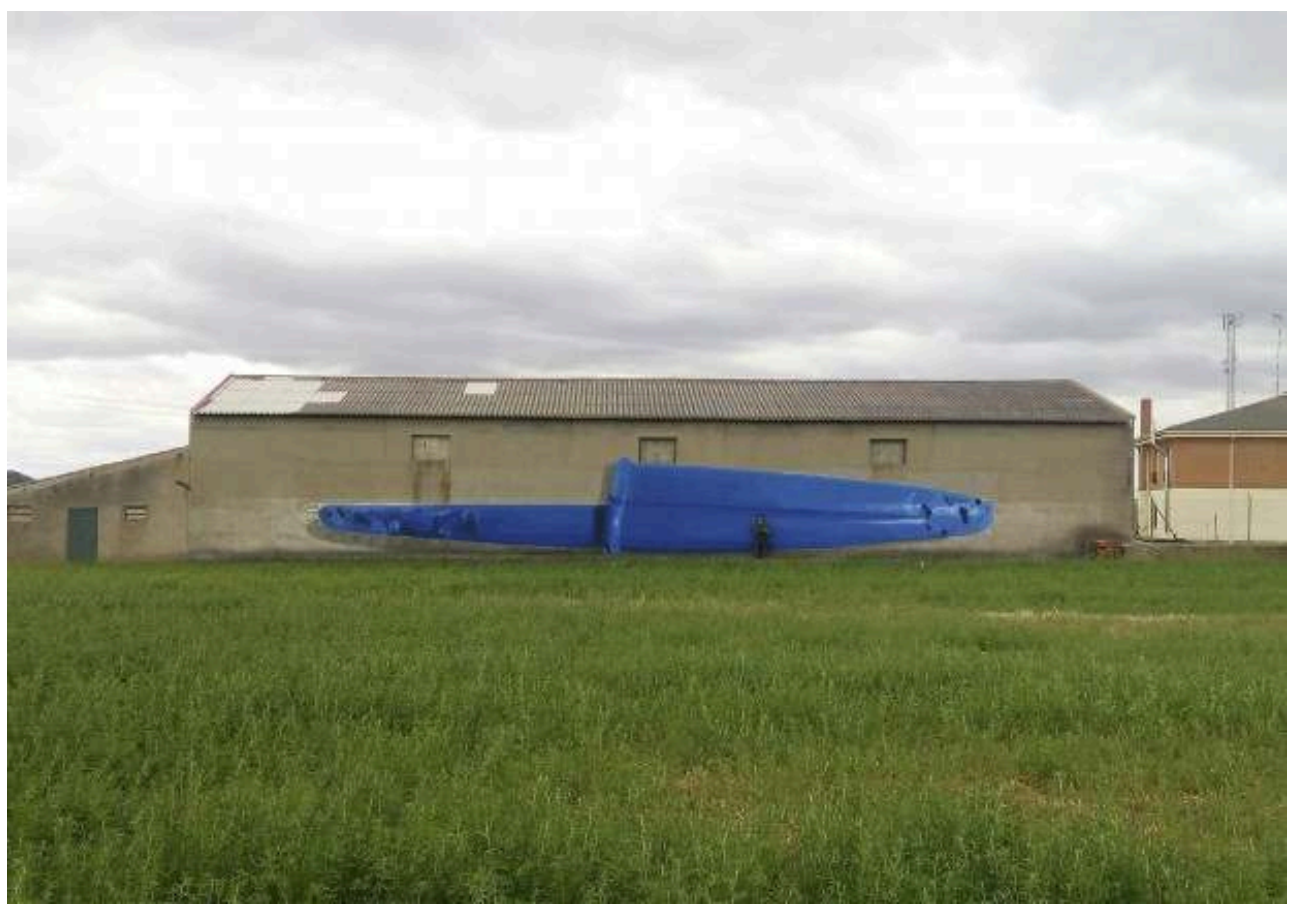

(C) Ampparito

Si l'on regarde attentivement l'objet, on verra qu'Ampparito a poussé le réalisme au point de représenter les mordillements dus, bien souvent, à un état de stress du propriétaire du stylo. Sur sa page Instagram, les explications qu'il fournit mêlent de nouveau autodérision et démarche introspective :

Mi círculo social y yo estamos chapoteando en un cóctel hecho de ansiedad, incertidumbre, presión social y rechinar de dientes desde hace unos meses, además Madrid está lleno de cosas alergénicas, calor seco y gente con prisa. Por esas razones decidí ir al campo y poner una tapa de bolígrafo azul masticada de 15 metros en un edificio, propiedad de un jubilado que no entendía lo que estaba pasando ${ }^{6}$.

Ampparito commente ses œuvres, les accompagne de ses mots. Il explique à chaque fois, sur les réseaux sociaux, comment lui est venu l'idée et dévoile ses mécanismes intellectuels. Cette démarche traduit une volonté de rester accessible et de ne pas jouer le rôle du créateur hermétique, inaccessible et/ou incompris. Cela lui permet aussi de prendre du recul sur sa pratique et d'adopter un regard distancié et parfois ironique.

Ce bouchon de stylo pourrait matérialiser l'état d'esprit d'une partie de la jeunesse espagnole, à laquelle il ferait allusion à travers ces mots " Mi círculo social y yo ». On pourrait envisager ce stylo comme une représentation d'un groupe social à peine sorti des bancs de l'école et préoccupé par la société dans laquelle il évolue. On pourrait aussi y voir une référence au parcours de l'artiste lui-même. Le mur apparaitrait comme un écran où viendraient se projeter les angoisses d'un débutant, qui n'est vraisemblablement pas issu d'un milieu artistique et qui a décidé d'embrasser la profession de peintre dans un contexte politique et économique peu engageant. Peindre sur les murs constituerait une échappatoire salutaire, un procédé qui lui permettrait de prendre de la distance sur sa pratique artistique. Il a conscience, par 
ailleurs, que ses envolées ironiques peuvent décontenancer les spectateurs et, en particulier, le propriétaire du mur auquel il fait allusion dans ses explications.

Sous les pinceaux d'Ampparito, le mur acquiert de nouvelles fonctions. Les œuvres imposantes qui le parent font oublier ses attributions matérielles et son caractère limitrophe. Ampparito reprend les codes dadaïstes et propose une réinterprétation d'éléments prosaïques. Il s'amuse à faire naître des réminiscences chez le spectateur. Il faut y voir sans doute une volonté de le faire sourire, mais pas seulement. Des détails troublants pourront en inciter certains à réfléchir à la charge symbolique de ces objets. De ce fait, l'espace intime, privé, est activé par ces interventions déroutantes et la frontière entre espace public et espace privé devient floue.

\section{La fonction ludique du mur}

Nous l'avons vu, l'humour est au centre du processus créatif de l'artiste. On peut de nouveau le constater avec l'intervention réalisée, un an après, à Saragosse, intitulée Ejercicio de bajar metro y medio un muro de un parque.

Figure 3. Ampparito, Ejercicio de bajar metro y medio un muro de un parque, Saragosse, 2017

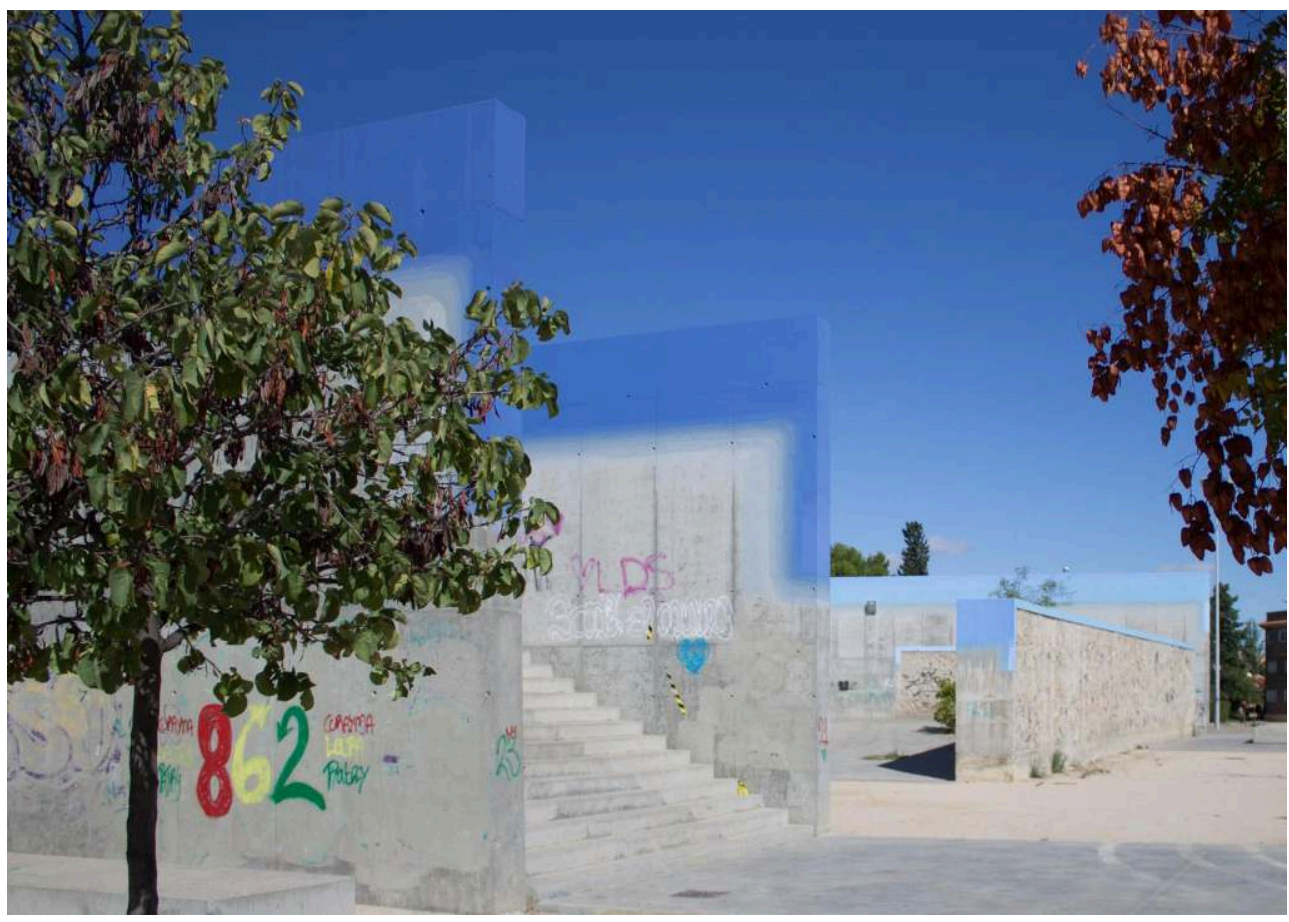

(C) Ampparito

Son travail a consisté à donner l'illusion qu'une partie des murs de ce parc de Saragosse disparaissait, en façonnant sa palette de bleu afin de pouvoir se rapprocher au maximum de la tonalité du ciel d'été de Saragosse. Il est revenu assez furtivement sur sa démarche :

Acabo de recibir estas fotos de la que ha sido mi intervención más extraña hasta el momento, desenfocando y eliminando un metro y medio cada pared en el parque más feo de Valdefierro, Zaragoza, para el festival Asalto ${ }^{7}$. 
Ses mots nous aident à comprendre, en partie, la genèse de cette œuvre : ce parc bétonné est, en effet, peu engageant. Habituellement arborés et pourvus de bancs, les parcs sont des espaces de sociabilité offrant des parenthèses au sein des villes ou des villages. Ils permettent que s'y développent des activités liées à la détente, au sport ou au jeu. Tel qu'il est conçu, ce parc n'offre que très peu d'éléments naturels, d'où la proposition d'Ampparito de laisser davantage de place au ciel. Le trompe-l'œil fonctionne et le mur s'estompe sous les pinceaux consciencieux d'Ampparito, qui de ce fait, endosse le rôle d'architecte. Avec sa proposition, le peintre introduit le jeu dans cet espace austère, celui de faire disparaître cette barrière superflue sans toutefois la détruire.

À propos d'aire de jeu, il a réalisé, peu de temps après, une marelle. Comme toujours, son intervention désarçonne et redéfinit la fonction du mur : ici, il ne constitue plus une limite mais un prolongement. Ampparito a voulu inviter les enfants à se servir de cette marelle, dont il a modifié les règles, pour créer « des happenings, des situations inattendues, métaphoriques ", a-t-il expliqués.

Figure 4. Ampparito, Sobre Rayuela, límites y los 30, Aviá, 2018

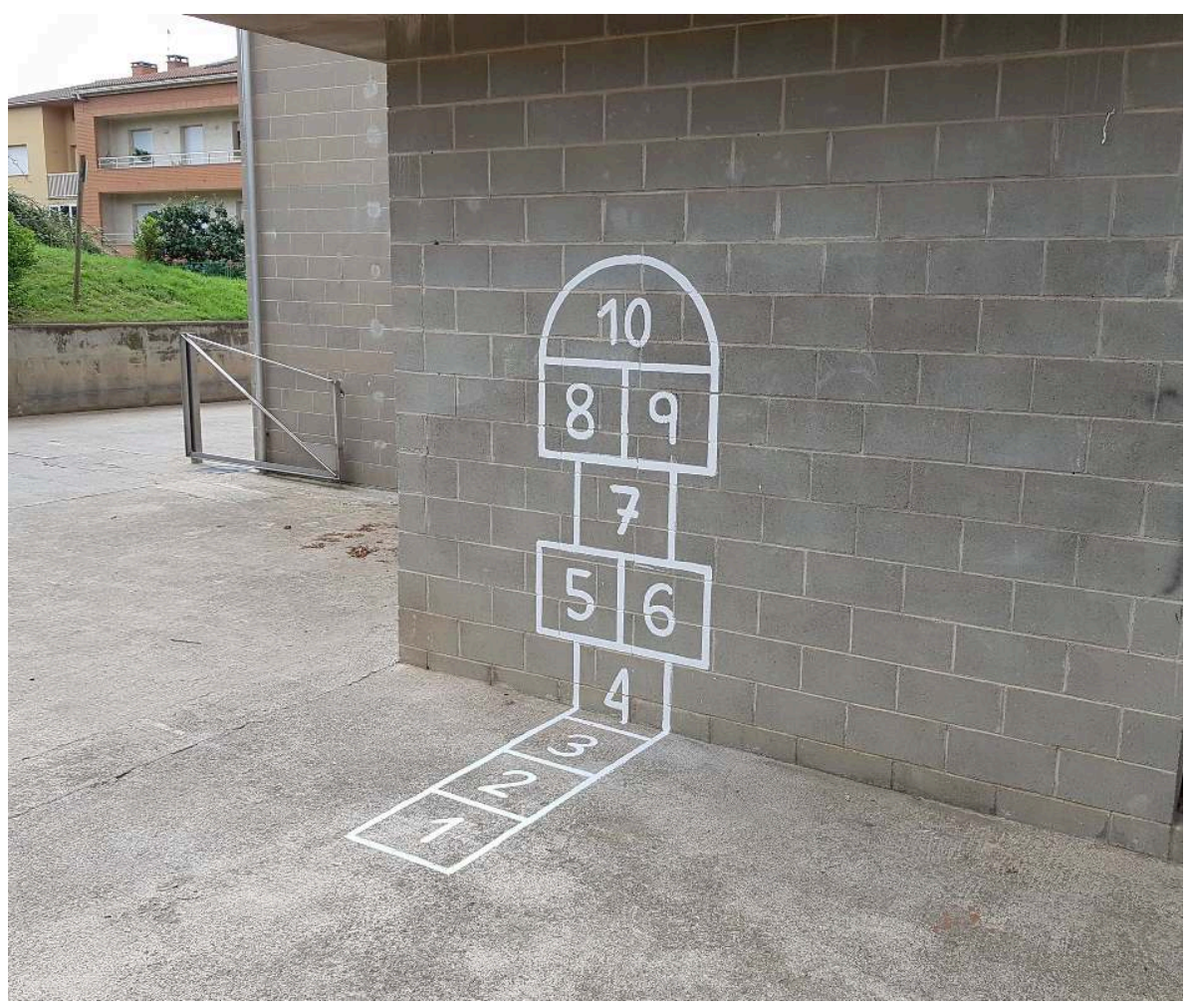

(c) Ampparito

À partir de 2018, il cherche à mettre en place des interventions qui permettent l'interaction directe avec le public. Et le mur, incarnant habituellement une limite, devient avec Ampparito, un support complice de ses détournements, une fenêtre ouverte sur l'imaginaire :

Creo que esto es interesante porque el público, la mayoría niños, no tienen ningún problema en usarlos pero cambiando las reglas para adaptarse a las nuevas características, dando pie a extrañas situaciones, algo así como performances. Estos 
juegos funcionan como activadores de inesperadas situaciones altamente metafóricas ${ }^{9}$.

Il cherche à intégrer le spectateur de différentes manières, à la fois physiquement et intellectuellement. En tant qu'interaction physique, parce que l'artiste crée des situations invitant à la performance, il tend des perches pour que le spectateur s'approprie à sa façon le terrain de jeu proposé. Ensuite, parce que cela permet de mobiliser la réflexion du passant et de lui faire vivre une expérience esthétique soudaine et inattendue.

La présence croissante d'œuvres picturales dans la rue n'a pas bouleversé les attentes des passants: on continue d'arpenter les rues pour aller d'un point à un autre, non pour contempler une œuvre d'art. Pour qu'il y ait rencontre entre une œuvre d'art et un individu, il faut généralement que ce dernier en fasse la démarche. Lorsque l'inverse se produit, que le rapport entre objet et humain est inversé, que l'image surgit sur un trajet quotidien, qu'elle s'interpose entre un individu et le chemin qui le mène au travail et semble animée d'une intention malicieuse, un mécanisme s'enclenche dans la tête du récepteur. La surprise créée par une œuvre hors de son espace sacré va forcer le spectateur à la prendre en compte et initier une forme d'interaction. En somme, comme l'expliquent Antonio Casado et Ana Liñero, "se consigue eso tan difícil en nuestros días: que el transeúnte de los espacios públicos se detenga a pensar por un momento $»^{10}$.

Cette volonté de bousculer les règles, il va la prolonger dans une série d'interventions visant à reproduire des espaces de jeu collectif. Il va ainsi proposer de nouvelles règles à des jeux très ancrés dans l'imaginaire collectif.

Figure 5. Ampparito, Sobre límites, espacio y pelota, Aviá, 2018

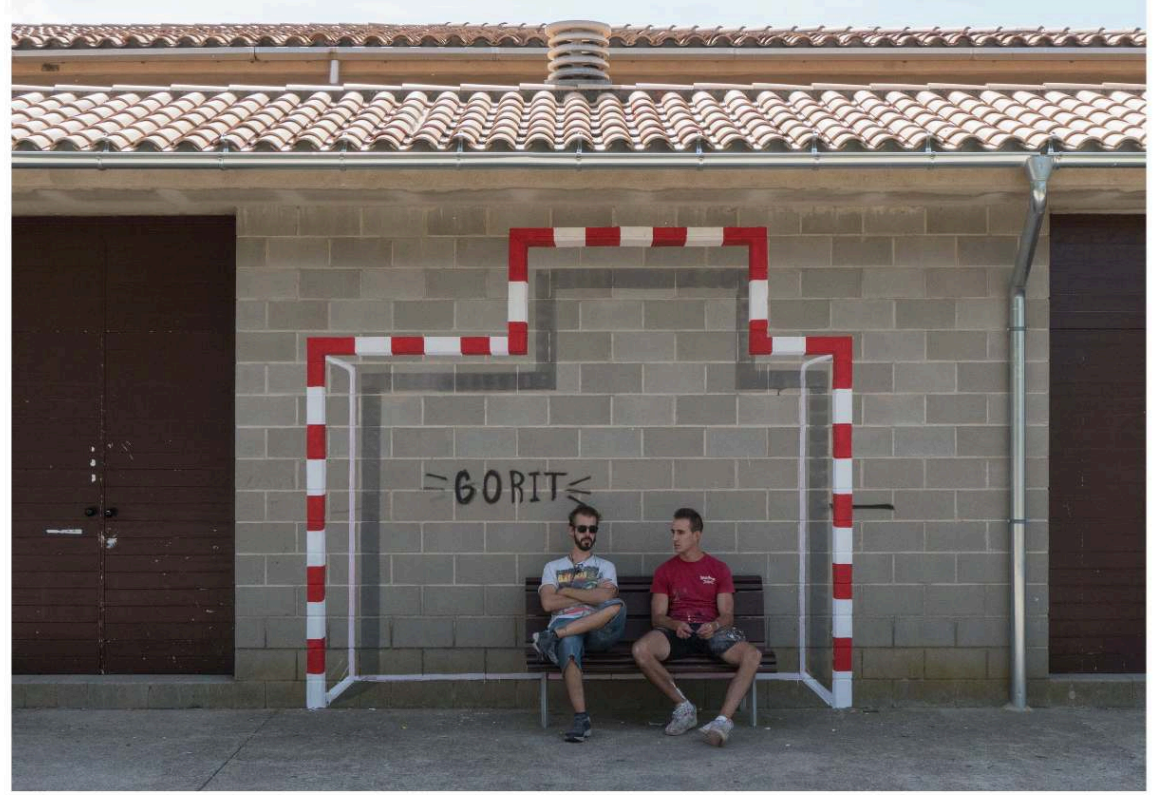

(C) Ampparito

Commençons par observer ce but de football, qui fait partie d'une série qu'Ampparito a réalisée en Espagne. On peut constater, tout d'abord, la fonction performative de ces peintures. Dès lors qu'elles sont apparues, il est sûr à $100 \%$ que le mur s'est réellement transformé en but. Par ailleurs, il y a également de fortes probabilités pour que les 
joueurs se soient adaptés à la nouvelle physionomie de ces buts et l'aient acceptée comme un défi, les invitant à viser avec précision. Enfin, peut-être peut-on voir dans ces appendices irréguliers un clin d'œil à la mauvaise foi enfantine (ou adulte) qui consiste à soutenir que le but " y est ", alors qu'en réalité, le ballon est passé à un mètre au-dessus de la ligne imaginaire dudit but.

Ces variations sur le thème du jeu collectif ont également inclus le basket. Ampparito a joué avec la fenêtre visant, en principe, à faciliter le tir. Dans cette série d'interventions sur le basket, il brouille les pistes et désoriente les joueurs. En outre, son approche offre une réinterprétation du mobilier urbain sportif, communément répandu dans l'espace public. Ces panneaux deviennent des éléments graphiques qui acquièrent une triple fonction, à la fois sportive, ludique et esthétique.

Pour illustrer cette série, nous pouvons donner l'exemple de cette intervention, qui pourrait s'intituler «Le basket pour les nuls ", mais qui, en réalité, a pour titre Sobre hierba verde, ladrillos naranjas, cielo azul y un tablero horizontal sobre un edificio amarillo descuidado.

Figure 6. Ampparito, Sobre hierba verde, ladrillo naranja, cielo azul y un tablero horizontal sobre un edificio amarillo descuidado, Villalán de Campos, 2019

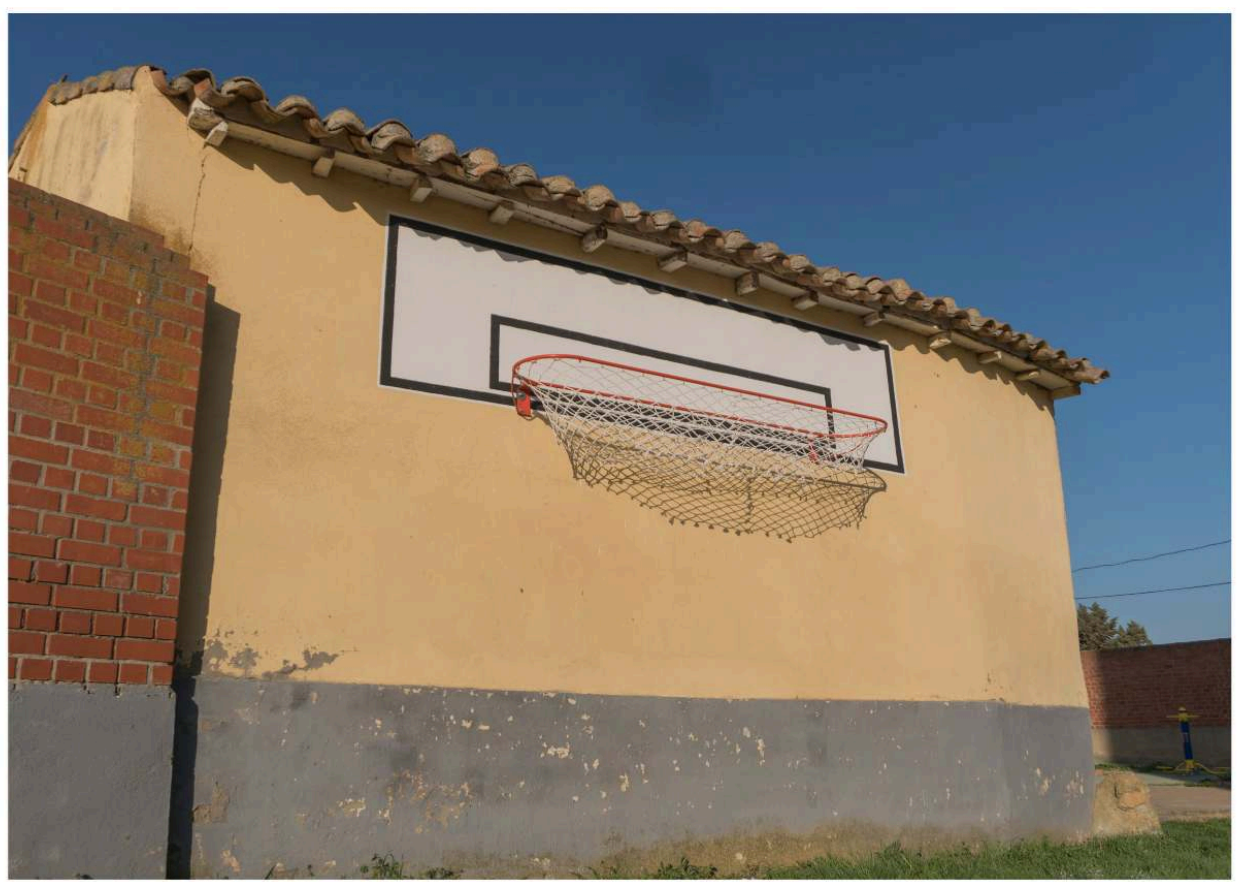

(c) Ampparito

Sa proposition déforme le panier de façon à ménager un maximum la susceptibilité des joueurs. Il est intéressant de constater comment Ampparito crée une mise en abyme du concept de jeu, en intégrant la notion de jeu dans le jeu :

La parte buena cuando reproduzco elementos muy conocidos popularmente en la calle, como pueden ser señales, porterías, campos de futbol, aros de baloncesto, rayuelas, etc; es que la mayoría de la gente piensa que no es nada relacionado con el arte, por lo que normalmente es visto como la consecuencia de un vago o chapucero operario. Este punto de comienzo es genial porque funciona como activador para potenciales performances, creadas, sin saberlo, por las personas que usan esos juegos modificados ${ }^{11}$. 
Nous terminerons cet article avec une intervention réalisée sur le mur d'une prison de Barcelone, sur lequel il a peint des prises d'escalade.

Figure 7. Ampparito, Sobre las prisiones, los muros de escalada y la imposibilidad de crear sombras con la pintura, Barcelone, 2018

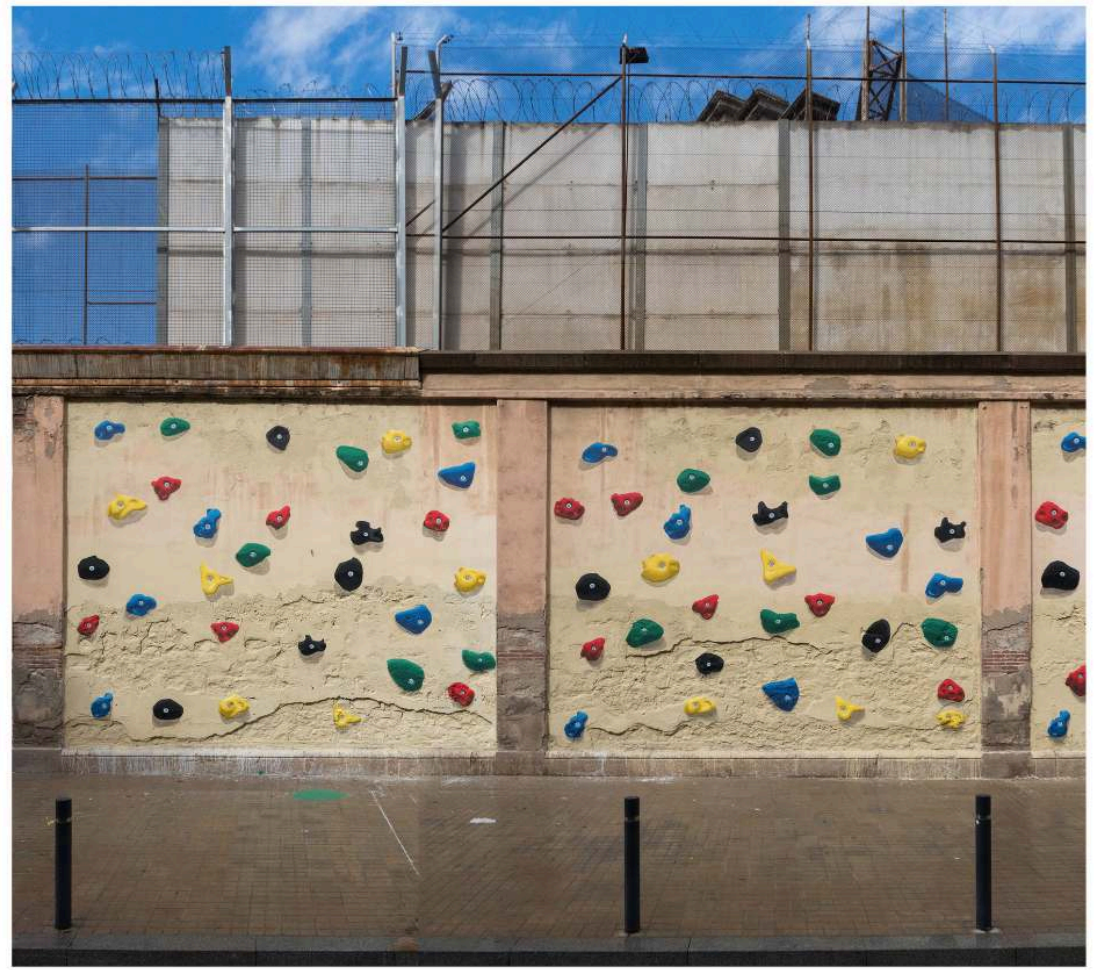

(c) Ampparito

Au-delà de la référence ironique à l'échappatoire, il propose une nouvelle forme de mise en abyme, linguistique, cette fois-ci :

Otro punto interesante es que en español estas presas de escalada se llaman "presas", literalmente el femenino de "presos" que significa prisioneros. Como toda la población de esta prisión eran hombres era común ver largas colas de mujeres esperando para visitar a sus maridos, hermanos o hijos, de alguna manera ellas, personas libres, estaban atadas a estos muros por culpa de sus hombres. Esto podría verse como un homenaje a estas mujeres también "presas" por "presos"12.

\section{Conclusion}

Ampparito offre une vision assez transparente de son processus créatif, en ce qu'il explique volontiers la genèse de ses œuvres. Malgré l'apparente simplicité de sa démarche et la banalité des sujets qu'il peint, ses interventions sont assez complexes. Nous avons constaté, pour commencer, que le mur pouvait accueillir une part de nondits. Ce qui permet à chaque spectateur d'apporter sa propre lecture. D'autre part, nous avons avancé l'idée de porosité entre espace public et privé. La fonction première du mur limitrophe semble, en effet, atténuée. Mais la complexité du travail d'Ampparito réside aussi dans ces variations autour du concept de jeu : les murs qu'il occupe, liés au quotidien, à une forme de prosaïsme, de barrière primaire entre l'espace des uns et des autres, deviennent des espaces de jeu, destinés à la fois aux usagers, mais aussi à 
l'artiste lui-même qui s'amuse de voir ses œuvres utilisées par les spectateurs. Ampparito désempare dans un jeu spéculaire qui consiste à tisser des histoires en laissant le hasard des rencontres faire les choses. Sans pour autant annuler le rôle premier du mur, son travail pictural dans l'espace public ajoute une dimension ludique et performative, modifiée à son tour par l'attitude des passants face à son œuvre.

\section{BIBLIOGRAPHIE}

Javier ABARCA (2009), « Arte urbano » [on-line], Madrid, Urbanario, [consulté le 14 avril 2021] <URL : http://urbanario.es/>.

Javier ABARCA (2016), « Del arte urbano a los murales, ¿qué hemos perdido? » [on-line], Madrid, Urbanario, [consulté le 14 avril 2021] <URL : https://urbanario.es/articulo/del-arte-urbano-a-losmurales-que-hemos-perdido/>.

Enrique ALPAÑES (2020), « Ampparito ha hackeado tu ciudad » [on-line], Madrid, YOROKOBU, [consulté le 14 avril 2021] <URL : https://www.yorokobu.es/ampparito/? fbclid=IwAR3zrpBmGoTcTFX4Osz y6gY33LlGhMnPNdloXDZ_rrqR6yoSkzUjmr2ZZY >.

María ARRANZ (2019), « Los muros pintados de Europa » [on-line], Madrid, El País, [consulté le 14 avril 2021] <URL : https://elpais.com/ccaa/2019/01/27/madrid/1548610362_658460.html? fbclid=IwAR16GPbfyu3MVFHDUzoV-r4rIFAKRuRVP90VLpkTq0N_UamtfbaPIrAUDqk>.

Antonio CASADO DA ROCHA et Ana LIÑERO REGLERO (2016), « La práctica caminante-desobediente como arte público independiente » [on-line], Madrid, Efimera Revista, [consulté le 14/04/2021].

Karla CORAzón (2018), « Ampparito, ya te vale » [on-line], México, All city canvas, [consulté le 14 avril 2021] <URL : https://www.allcitycanvas.com/ampparito-en-entrevista-por-edunose>.

\section{NOTES}

1. Enrique ALPAÑES (2020), «Ampparito ha hackeado tu ciudad», [on-line], Madrid, YOROKOBU, [consulté le 14/04/2021] <URL:https://www.yorokobu.es/ampparito/? fbclid=IwAR3zrpBmGoTcTFX4Osz y6gY33LlGhMnPNdloXDZ_rrqR6yoSkzUjmr2ZZY >.

2. " tags con gancho, nombres molones, guerra de estilos ", Ibid.

3. Javier ABARCA (2009), «Arte urbano» [on-line], Madrid, Urbanario, [consulté le 14 avril 2021] $<\mathrm{URL}:$ http://urbanario.es/>.

4. « pillar desprevenido, de descolocar », in Enrique ALPAÑES, op. cit.

5. Les réseaux sociaux constituent un outil précieux pour comprendre le travail d'Ampparito, qui commente ses interventions sur ses pages Facebook et Instagram: <URL: https:// www.facebook.com/ampparitos/> et <URL: https://www.instagram.com/ampparito/>.

6. La citation est tirée du compte Instagram d'Ampparito, [consulté le 14 avril 2021] <URL: https://www.instagram.com/p/BG3lSFOGnDD/>.

7. La citation provient de son compte Instagram, [consulté le 14 avril 2021] <URL: https:// www.instagram.com/p/BeSi5qcgx7_/>. 
8. On peut retrouver l'intégralité de sa citation ici [consulté le 14 avril 2021] : <URL : https:// www.instagram.com/p/BniptEolxyH/>.

9. La citation pourra être consultée sur cette page Instagram: <URL: https:// www.instagram.com/p/BniptEolxyH/>.

10. Antonio CASADO DA ROCHA et Ana LIÑERO REGLERO (2016), «La práctica caminante-desobediente como arte público independiente » [on-line], Madrid, Efimera Revista, [consulté le 14/04/2021].

11. La citation peut être lue sur la page Facebook d'Ampparito [consultée le 14 avril 2021] <URL : https://www.facebook.com/ampparitos/posts/2128011820588527 >.

12. Ibid.

\section{RÉSUMÉS}

Ampparito se présente sur son site comme un artiste qui : «travaille à subvertir des objets, des significations et des réalités pour générer de nouvelles expériences ou situations. Celles-ci peuvent donner lieu à une vaste gamme de résultats, allant de l'indifférence la plus absolue à la réflexion la plus profonde, en passant par la désorientation ou la contemplation ». Le lecteur qui aura pris le temps de lire ces quelques mots saura déceler la malice et la dissonance avec lesquelles l'artiste conçoit ses interventions. Adepte du trompe-l'œil et du détournement, Ampparito embarque le passant dans ses délires ludiques jusqu'à en faire oublier complètement le mur qui les accueille. Dans cette perspective, le mur acquiert une autre fonction, « impliquant ou créant la dynamique de son dépassement». Nous proposons d'étudier les interventions laissées par Ampparito dans l'espace public espagnol. À l'heure de la consolidation des frontières et du repli sur soi, s'amuser du mur tel que le fait Ampparito reviendrait-il à montrer la brèche possible?

Ampparito presents himself on his website as an artist who: "works to subvert objects, meanings and realities to generate new experiences or situations. These can give rise to a wide range of results, from the most absolute indifference to the most profound reflection, through disorientation or contemplation". The reader who takes the time to read these few words will be able to detect the malice and dissonance with which the artist conceives his interventions. An adept of trompe-l'œil and diversion, Ampparito embarks the passer-by in his playful delirium to the point of making him completely forget the wall that hosts them. In this perspective, the wall acquires another function, "implying or creating the dynamics of its overcoming". We propose to study the interventions left by Ampparito in the Spanish public space. At a time when borders are being consolidated and people are turning in on themselves, is having fun with the wall, as Ampparito does, the same as showing the possible breach?

\section{INDEX}

Mots-clés : street art, art public, subversion, détournement, Espagne, Ampparito

Keywords : street art, public art, hacking, hijacking, Spain, Ampparito 
AUTEUR

ANNE PUECH

Université de Rennes 2 